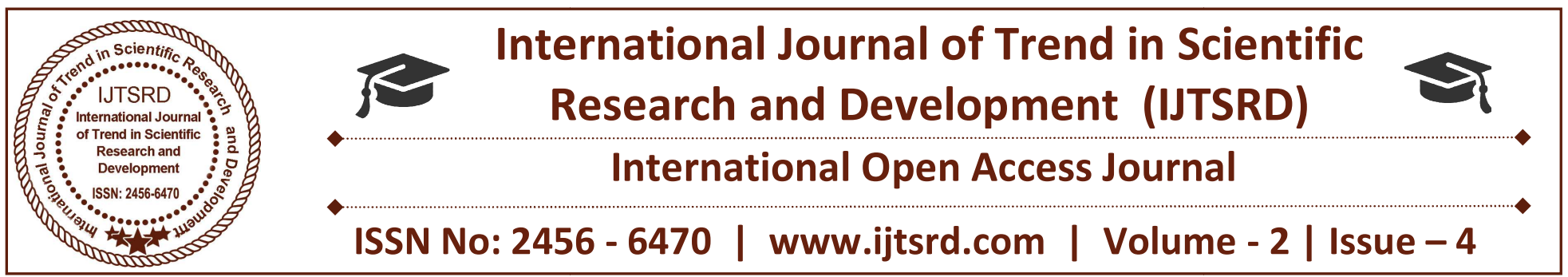

\title{
MED-CARE Using IoT
}

Aishwarya C, Ankita Vyas

Department of Information

Science and Engineering

School of Engineering and technology, Jain University, Bangalore, India

\section{Sunil M P}

Department of Electronics and Communication Engineering, School of Engineering and Technology, Jain University, Bangalore, India

\author{
Manjunath C R \\ Department of Computer Science \\ and Engineering, \\ School of Engineering and \\ Technology, Jain University, \\ Bangalore, India
}

\begin{abstract}
Many high-risk diseases can be medicated and prohibited with the use of right kind of treatment. Accordingly, it's compulsory to consume drugs on the recommended time. If an infirm doesn't consume his medicant on schedule, there may be various negative effects. Using medical science and technology, this methodology assures specific time alert for the intake of the medicants. The alert will be one in audio form and other in visual form. Audio alert in the form of buzzer and visual alert in the form of LED and WhatsApp message. This device helps the infirm and the physician to monitor the regular intake of medication and advice for better improvements of the health.
\end{abstract}

Keywords: Internet of Things (IoT), Raspberry Pi, Alert, Reed Switch

\section{INTRODUCTION}

The Internet of Things (IoT) is the mesh of physical devices, machines and other items installed with electronics, sensors and connectivity which enables these objects to connect and exchange data. "Things" in IoT refers to a wide diversity of instruments alike disease examining implants, automobiles with built-in sensors, DNA analysis device for environmental or food or pathogen monitoring and so on. IoT is creating more opportunities for direct integration of physical world into computer based system which leads to less human intervention. IoT results in improved efficiency, accuracy, economic benefits and when IoT is raised with actuators and sensors it encompasses technologies such as smart cities, intelligent transportation, smart homes, remote health monitoring system. IoT device collect useful data with the help of various existing technologies and then autonomously flow the data between the devices.

Iot in health care is a miscellaneous computing, cordlessly communicating system of devices and apps that binds patient and health providers to monitor, store, and track and diagnose vital stats and medical data. IoT in medical and healthcare field can be used to enable remote health monitoring system and emergency notification systems. The major advantages of IoT in healthcare organizations are: Improved outcome of treatments. Connectivity of healthcare with IoT gives the people the capacity to retrieve real time information that permit them to perform informed decisions to provide therapy that is evidence based. This insures health protection provisions are punctual and treatment outcomes are improved. Better disease administration. When infirm's are observed on a continuous basis and health protection providers are able to retrieve real time information, diseases are treated before they become turbulent. Augmented infirm's Experience. The connectivity of the health protection system via internet of things, places prominence on the needs of the infirm. 


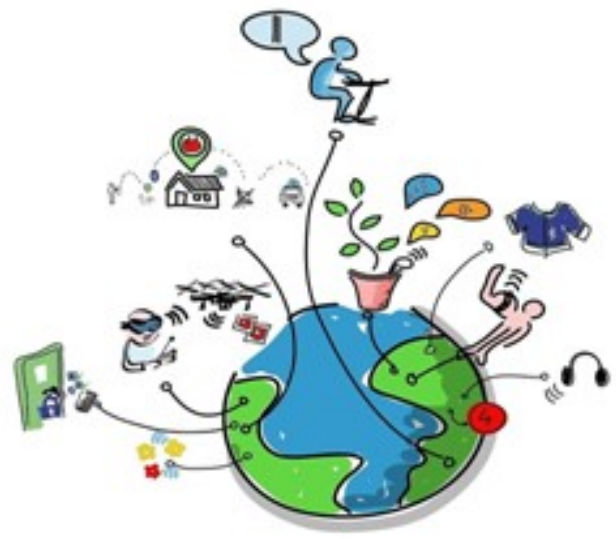

Fig. 1: Internet of Things

That is, dedicated treatments, improved correctness when it comes to detection, well timed intervention by specialists and augmented treatment conclusion result in responsible care that is immensely trusted among infirms. Augmented Management of Medicines. Production as well as management of medicines is a important outlay in the healthcare corporation. Even then, with IoT devices and procedures, it is likely to manage these expenses better. Immediate medical awareness especially during phase of medical crisis and natural disaster. Eliminates the need to physically go to a medical facility. Expanded reach to various health service providers.

Medication should be taken regularly to make sure that the body has an effective amount of drug in the body at all times. If a infirm does not take his medicant on day to day basis, there can be certain negative effects such as the level of drug in the blood can become less effective to stop the viruses from multiplying. This gives the virus a chance to mutate and become resistant in the blood.

Pills are the fundamental results for prohibition and remedy for most of the diseases. Many dangerous diseases can be prohibited and medicated with the right kind treatment. For this reason, it is required to consume the pills at the recommended time.

People in today's world are so busy and engaged in work that they forget even to eat food. There are many chances to forget their medication along with it which affects them in various ways. There are many chances to fall sick frequently or die. Elderly person due to their age and loss of memory forget to take medication on time or when the patient is alone at home and has fallen down due to some sickness, the person cannot help himself. In this world, using technology and internet many things are invented to address the problems for the current situation, but it cannot always be the solution. As the time passes, the same problem has to be addressed in different ways.

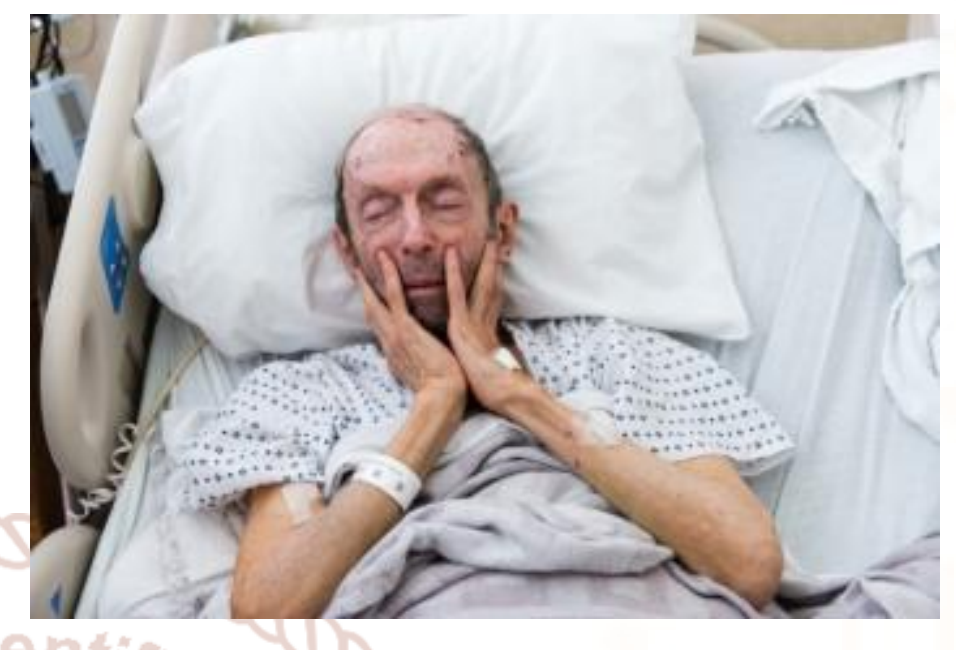

Fig. 2: Elderly patient

Using IoT, different systems or devices were developed to help patient so that they take medication on time. The device that notifies the patient to take medicines, but the patient ignores it due to their busy schedule or forgets it.

The med-alert device was designed to alert the patient to take medication on time. The Raspberry Pi 2 model $\mathrm{B}$ was used to design this device. The similar device can be designed using different versions of Raspberry $\mathrm{Pi}$, Arduino, GSM and other embedded systems.

The sick person can use as a remainder alert for the intake of day to day medicants on time. This system make sure that provides specific time alert for the intake of the pills. The device alert was a buzzer that beeps for certain interval of time and LED is turned on until the patient opens the box. After an interval of time if the patient still does not open the box, the alert is sent to other members of the family via e-mail. There are many chances that the person or other family members not open the e-mail immediately and forgets about it. Else there are many chances of the person to just open and close the box without taking the medication so the LED is turned off. This again is a problem of missing the medicines.

To address this problem, the med-alert device is improvised to send an alert message via WhatsApp which every person is familiar with and checks the application frequently. First, when the device alerts the person through a WhatsApp message, then the buzzer beeps and the LED is turned on. Ever after certain duration the person does not take then 
medicine, the device alerts other family members about it. The person cannot just open and close the box to avoid the LED. Until the patient takes his medication the LED remains on. This is monitored by the weight sensors inserted in the device, until the weight of the box is not reduced, LED remains on. So the patient's regular intake of medicines can be monitored easily.

\section{THE EXISTING WORK}

People are vulnerable to diseases of alternative types because of their busy schedule, responsibilities and stress. It is our commitment to keep ourselves fit and in good physical condition. The extraordinary issue is that infirms fail to remember to consume appropriate medicine in prescribed time. Medication obedience, that refers to the grade or measure to which a victim takes the correct medicants on the correct time as prescribed by specialists, has lately come out as a threatening business due to few studies have described that omission may extremely influence the victim, with raising medicinal costs. Medication nonadherence is a common, complicated, and expensive issue that leads to weak medication results and consumes medicinal management sources [4].

An Android application whose purpose is to assist the victims to remember their prescription timings over Alarm Ringing system in order that they take medicines in proper time. Proper treatment can be given on time by searching doctors, hospitals and contact details through navigation. In this application user can set an alert along with fields of date, time and medicine description that will let them to set alarm for couple of medicines at various interval of time. Warning is sent after setting an alarm. The application is designed on Eclipse. It may be favorable in defense sector and emergency circumstances (accidents) and may circulate health protection alertness. It is lifesaving, efficient and handy application that is convenient and provides a great user interface [2].

The planned process is rest on Android Operating system that will note the users to take pills without delay over notification and automated alarm ringing process. The system is specified on android

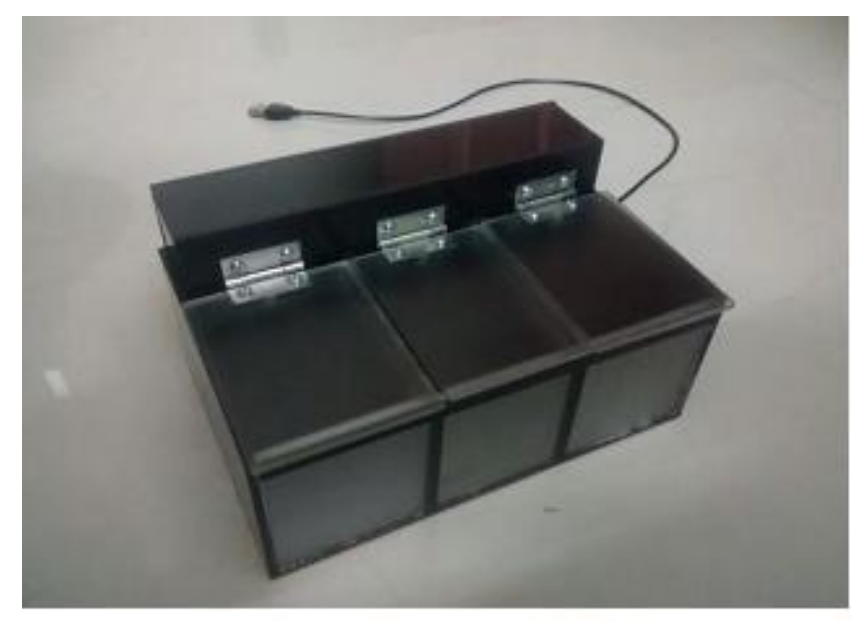

Fig.3. Medication case

operating system only because the market share of Android is high. The groundwork is designed to understand the rework and combination of components. Android apps are made having an imperative XML manifest file. The manifest file values are bound to the application at compile time. This file provides a vital report to an Android platform for dealing with the life cycle of an application. Examples of one's type of information constituted within a manifest file are descriptions of one's app components in connection with architectural and configuration properties. Components can be one of the following types: Activities, Services, Broadcast Receivers, and Content Providers.

Medication remainder system consists of setting alarm and getting notification where while setting the alarm the user can add on the description of medicine including purpose, name and also the starting date and ending date of the dosage between which the user has to take medicine as prescribed. Once the alarm is set the user gets the warning which the user can activate or deactivate accordingly [5].

Many medicinal systems has been evolved based mostly upon various platforms and ideas. Many healthcare apps happen to be advanced, but there are various problems associated with their performance and working. Based on the time and kind of medicine a warning will be sent in the form of email or message to the infirm as a reminder. An IoT device that was developed to alert the patient to take medicines on time. The device was designed in such a way that when it is time to take medicines it would send an alert Email to the user, for the audio alert buzzer beeps and for the visual alert LED is used as a remainder. 
In spite of these, if the user doesn't take pills on time, a remainder mail is forwarded to the other members of the family, so to alert the infirm to take pills. The Raspberry $\mathrm{Pi}$ is hooked up to the net through the Ethernet LAN cable. The net connection is initially required to renew, develop and $\log$ in vital packages for the Raspberry Pi. The Raspberry Pi's GPIO proceed the LED's which is necessary to light up the correct tablet that is to be taken at the prescribed time. The buzzer is also connected to the Raspberry Pi's GPIO. The buzzer simulates an audio notification to alert the infirm to take the specific medicant. When a victim takes the specific medicines, it's essential to notice their act. This might be accomplished by sensing the opening and closing of the box by allocating a reed switch and an adequately strong magnet. A substitute solution is to use magnetic sensors in place of the reed switches. The mail notification will be sent to any number of e-mail accounts identified. The default mail note is sent to the family members and infirm. Therefore, it is important for the mobile devices to have access to the internet.

Fig.3. depicts the medication case with three compartments. Each compartment is assigned for morning, afternoon and night with respective medicines in it. The device checks constantly if the current

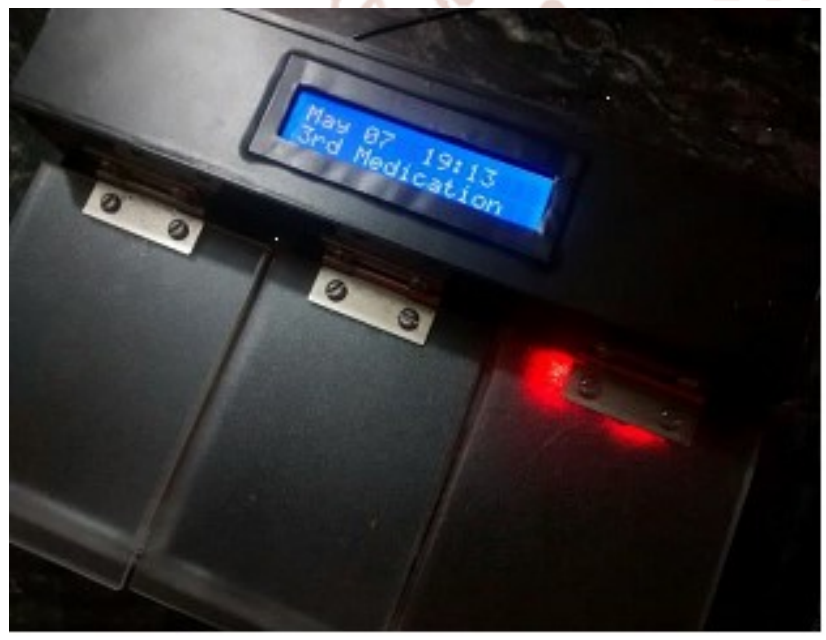

Fig. 4. Medication alert

time is same as the digitized time to notify the user about the consumption of tablets. If both the time matches, it immediately lights up the LED of the particular compartment and the buzzer begins to beep for absolute span of time if the user is in the other room. The buzzer turns off after 5 minutes but the
LED remains on for 15 minutes if the pills are consumed.

The two situations that would occur if the medication is not taken. Firstly, the patient consumes all the medicants. The reed switch result is observed by Raspberry $\mathrm{Pi}$ to analyse if the box was opened to consume pills. If the box is opened and closed to take pills, the LED turns off and waits for the next programmed time. Secondly, if the patient is not in audible or visible range, the system sends an Email to the patient's account reminding him to consume medicants after 5 minutes. If the patient is unfit to take medicants or forgets to take medicants, the system waits for 15 minutes after an email is sent to the infirm's account. After the 15 minutes, the device instantly sends one more mail to the members of the family to remind the patient to take medicants.

LED's are connected in laterally to each compartments to improve illumination per compartment. Therefore any other end of one's resistor terminals of two LED's of a certain slot are used. There's a reed switch attached below the hinge per chamber of the device. The hinge allows to open and close the slot with ease. The reed switch has two end points and has no polarity. One terminal from all the reed switch are shorted hooked up to ground on the Raspberry Pi. The LCD display is used to display the date, time and the medication number.

\section{THE PROPOSED SYSTEM}

From the observation in the current functioning of the device, the following would be the ones which might cause some trouble to the patient or the user: The user can just open and close the box to avoid the buzzer and the LED without consuming the pills and escape from the alert. Often, there are many possibilities of the user and the other family members miss to notice the email sent. There are many chances that the user might be away from the device.

The proposed system aims at enhancing the medication case by sending an alert through the WhatsApp, inserting the weight sensors to the box by using the modern day technique IoT.

Using this IoT device which gives an audio alert and visual LED alert that helps the patient to take medicines on time. The device also sends a WhatsApp message to the user at the scheduled time, if the user misses the medication immediately, then device waits 
for few minutes before sends an alert to the other members of the family to remind the person.

The weight sensors are used to keep track of consumption of pills by the patient. As the patient consumes the medicines the weight of the box should go on decreasing so as to identify that the patient has taken the medicines. Until the weight reduces the LED light would be on. The user might forget to purchase medicines at the end of the period due to his busy schedule. Once the medication period is completed, it is notified to the user via WhatsApp message.

In this device, WhatsApp is installed on Raspberry Pi through which a message is sent to the user and other members of the family. The weight sensors are integrated with the medication box. WhatsApp is installed on Raspberry $\mathrm{Pi}$ : installation includes updating the packages and firmware. After updating, it prepares the system with the necessary components to Yowsup, downloads the library and installs it. After the installation, the device has to be registered to use WhatsApp. Later to use the application, create a file to save the credentials. Once this is done, WhatsApp alert is sent to the user.

The system architecture gives the brief idea about the working of the device. At the scheduled time, the buzzer beeps, LED is turned on and remainder is sent to the user via WhatsApp message. The buzzer beeps for certain duration of the time if the user has not noticed. The buzzer turns off after 5 minutes but the LED remains on for 15 minutes if the pills are not taken. After this interval of time, the device again alerts the user and other family members to remind the patient to take pills.

Once the user is alerted with the buzzer and LED, there are two scenarios in which the user opens the box and takes the pills then the buzzer stops, LED gets off and also weight of the box reduces which ensures that the medicine has been consumed by the user. The other scenario is that the user just opens the box to avoid the sound alert where the LED remains turned on for certain duration of time and the weight of the box remains the same which shows that the user has not

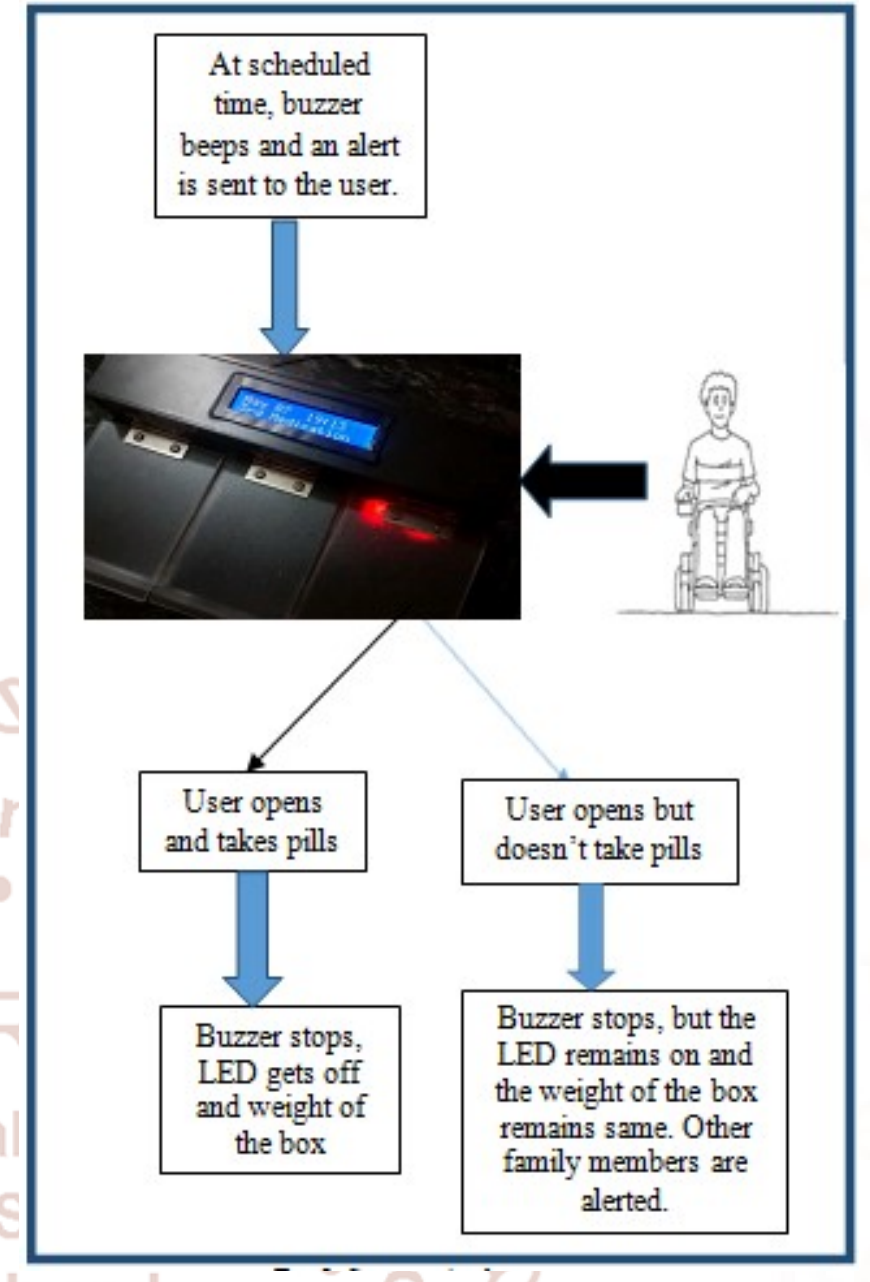

Fig.5. System Architecture

consumed the pills. The LED remains turned on until the weight of the box reduces and WhatsApp message is sent to the other family members after certain interval of time.

The benefits of the proposed work is that it user friendly, It helps the user to take med on time with the help of technology that everyone is aware of, as WhatsApp is being used by each and every person, it is easy to communicate with user which helps the user to be alerted, by giving a WhatsApp message to the user and other members of the family that the user have to take the medicine and if the user has not taken it the WhatsApp message is sent to the family members, which also helps the family members to be alerted about the patient and can take necessary actions. We are using weight sensors which helps in understanding whether the patient has consumed the medicine or not. Weight sensors measures the weight of the box, if the weight of the box has been reduced then the LED is turned off which shows that the user has consumed the medicines. 


\section{CONCLUSION}

In this paper, a feasible design has been proposed that the infirm can be wide awake to take their day to day medicant on time. This method insures specific time alert for the intake of the medication. The alert will be one in audio and the other in visual form. For easy detection and alert, buzzer and LCD display has been attached so that the person in concern takes the pills in time. For audio alert, buzzer is being used and LED is used for the visual form, LCD display that gives the details of the medication number, date, time and WhatsApp message has been sent. If the user is not consuming the pill even after the buzzer beeps, family members are alerted. Weight sensors are being used to sense that whether the patient has consumed the pills or not. Until the weight of the box gets reduced, the LED is turned on and message is sent after certain interval of time.

\section{REFERENCES}

1. Jayanth S, Poovi MB, Sunil MP "Med Alert-An IoT Device", 2016, International Conference on Inventive Computational Technologies (ICICT)

2. M Surya Deekshith Gupta, Vamsikrishna Patchava, Virginia Menezes, Healthcare based on IoT using Raspberry Pi, 2015 IEEE

3. Deepti Ameta, Kalpana Mudaliar, Medication Reminder And Healthcare-An Android Application, IJMPICT, Vol. 6, No. 2, June 2015

4. Mei- Ying Wang, John K. Zao, P.H. Tsai, J.W.S. Liu, Wedjat: A Mobile Phone Based Medication Reminder and Monitor

5. "Smartphone medication adherence apps: Potential benefits to patients and providers", available at:

6. http://www.ncbi.nlm.nih.gov/pmc/articles/PMC39 19626/

7. Mahmood R, Mirzaei N, Malek, S., (2014), "EvoDroid: Segmented Evolutionary Testing of Android Apps", FSE'14, November 16-21, 2014, Hong Kong, China

8. Samir V. Zanjal, Girish R. Talmale., (2015), "Medicine Remainder And Monitoring System Using IoT", ICISP

9. Shivakumar Chandrasekaran, Sathishkumar SenaMohan., 2016, "MAS-Med Alert System", 2016 IEEE $6^{\text {th }}$ International Conference on Consumer Electronics, Berlin
10. P. Raga Lavima, Mr. G. Subhramanya Sarma., "An IoT Based Intelligent Medicine Box", IJCSMC, Vol.4 Issue 10 October 2015

11. Jabeena, Rohit Roy, Animesh Kumar Sahu, N. Sadar Basha, 2017, "Automatic Pill Remainder For Easy Supervision”, ICISS IEEE Conference

12. Harshid Soni, Sanjay Bhati, Vijayrajsinh Zala, Parth Vyas, Mr. Yash Sharma, "Smart Medicine Box", International Journal of Science Technology and Engineering (IJSTE) 2017 Vol. 3 Issue 10

13. Aakash Sunil Salgia, K. Ganesan, Ashwin Raghunath, 2015, "Smart Pill Box", Indian Journal of Science and Technology (IJST). 